\title{
In vivo photoacoustic multi-contrast imaging and detection of protein interactions using a small near- infrared photochromic protein
}

Lei Li, Anton A. Shemetov, Peng Hu, Daria M. Shcherbakova, Junhui Shi, et al.

Lei Li, Anton A. Shemetov, Peng Hu, Daria M. Shcherbakova, Junhui Shi, Junjie Yao, Vladislav V. Verkhusha, Lihong V. Wang, "In vivo photoacoustic multi-contrast imaging and detection of protein interactions using a small near-infrared photochromic protein," Proc. SPIE 10878, Photons Plus Ultrasound: Imaging and Sensing 2019, 1087818 (27 February 2019); doi: $10.1117 / 12.2509198$ 


\title{
In vivo photoacoustic multi-contrast imaging and detection of protein interactions using a small near-infrared photochromic protein
}

\author{
Lei $\mathrm{Li}^{\mathrm{a}}$, Anton A. Shemetov ${ }^{\mathrm{b}}$, Peng Huc${ }^{\mathrm{c}}$, Daria M. Shcherbakova ${ }^{\mathrm{b}}$, Junhui Shi ${ }^{\mathrm{d}}$, Junjie $\mathrm{Yao}^{\mathrm{e}}$,
} Vladislav V. Verkhusha ${ }^{\mathrm{b}, \mathrm{f}}$ and Lihong V. Wang ${ }^{\mathrm{a}, \mathrm{d}^{*}}$

${ }^{a}$ Caltech Optical Imaging Laboratory, Department of Electrical Engineering, California Institute of Technology, Pasadena, CA 91125, USA; ${ }^{\mathrm{b}}$ Department of Anatomy and Structural Biology, and Gruss-Lipper Biophotonics Center, Albert Einstein College of Medicine, Bronx, NY 10461, USA;

${ }^{c}$ Department of Biomedical Engineering, Washington University in St. Louis, MO 63130, USA;

${ }^{\mathrm{d}}$ Caltech Optical Imaging Laboratory, Andrew and Peggy Cherng Department of Medical

Engineering, California Institute of Technology, Pasadena, CA 91125, USA; ${ }^{\mathrm{e}}$ Department of

Biomedical Engineering, Duke University, Durham, NC 27708, USA; ${ }^{\mathrm{f}}$ Medicum, Faculty of

Medicine, University of Helsinki, Helsinki 00290, Finland

*LVW@Caltech.edu;

\begin{abstract}
Photoacoustic (PA) computed tomography (PACT) is a non-invasive imaging technique offering optical contrast, high resolution, and deep penetration in biological tissues. PACT, highly sensitive to optical absorption by molecules, is inherently suited for molecular imaging using optically absorbing probes. Genetically encoded probes with photochromic behavior dramatically increase detection sensitivity and specificity of PACT through photoswitching and differential imaging. Starting with a DrBphP bacterial phytochrome, we have engineered a near-infrared photochromic probe, DrBphP-PCM, which is superior to the full-length RpBphP1 phytochrome previously used in differential PACT. DrBphP-PCM has a smaller size, better folding, and higher photoswitching contrast. We have also developed an advanced PACT technique, which combines the reversibly-switchable photochromic probes with single-impulse panoramic PACT, termed RS-SIP-PACT. Using RS-SIP-PACT, we have characterized DrBphP-PCM both in vitro and in vivo as an advanced near-infrared photochromic probe for PACT. We introduce two phytochromes into the same mammalian cells, resulting in a distinctive decay characteristic in comparison with the cells expressing DrBphP-PCM only. By discriminating the different decay characteristics, we successfully separate multiple cell types in deep tissues. The simple structural organization of DrBphP-PCM allows engineering a bimolecular PA complementation reporter, a split version of DrBphP-PCM, termed DrSplit. DrSplit enables PA detection of protein-protein interactions in deepseated mouse tumors and livers, achieving $125-\mu \mathrm{m}$ spatial resolution and 530-cell sensitivity in vivo. The combination of RS-SIP-PACT with DrBphP-PCM and DrSplit holds great potential for non-invasive multi-contrast deep-tissue functional imaging.
\end{abstract}

Keywords: Photoacoustic computed tomography, near infrared photoswitchable protein, multicontrast imaging, proteinprotein interactions

\section{INTRODUCTION}

To better understand the molecular mechanisms and dynamics involved in physiology and disease in a whole organism, biomedical studies increasingly employ noninvasive whole-body imaging with high resolution in vivo[1-3]. Optical imaging offers valuable information and has been widely used in such studies[4-7]. However, photons are strongly scattered in biological tissue, limiting high-resolution pure optical imaging to a penetration depth within the optical diffusion limit $(\sim 1 \mathrm{~mm})$ [8-10]. Photoacoustic (PA) computed tomography (PACT), by acoustically detecting photons absorbed by tissue, breaks the resolution and depth limitations of pure optical imaging and provides high resolution imaging with optical contrast at depths up to centimeters[11-14]. PACT, highly sensitive to optical absorption by molecules, is inherently suited for molecular imaging using optically absorbing probes.[15-18] Genetically encoded probes are advantageous due to their harmless non-invasiveness, precisely controllable targeting, and tissue-specific promoters. The combination of PACT and a reversibly photoswitchable near-infrared (NIR) absorbing full-length

Photons Plus Ultrasound: Imaging and Sensing 2019, edited by Alexander A. Oraevsky, Lihong V. Wang, Proc. of SPIE Vol. 10878, 1087818 - @ 2019 SPIE · CCC code: 1605-7422/19/\$18 · doi: 10.1117/12.2509198 
bacterial phytochrome (BphP) from Rhodopseudomonas palustris, RpBphP1, has resulted in an advanced differential imaging technique, termed reversibly switchable PACT (RS-PACT). RS-PACT provided substantially enhanced detection sensitivity in deep tissues[19] in comparison with conventional PACT.

Structurally, BphP proteins consist of a photosensory core module (PCM) and various so-called effector domains (Fig.1a). The PCM is formed by the PAS (Per-ARNT-Sim), GAF (cGMP phosphodiesterase/adenylate cyclase/FhlA), and PHY (phytochrome-specific) protein domains connected with $\alpha$-helix linkers, and typically has a molecular weight of 55-58 kDa. The spectral properties of BphPs are determined by a covalently attached chromophore, biliverdin IX $\alpha$ (BV). Canonical natural BphPs have two absorbing states, one of which absorbs at 670-700 nm (the Pr state) and the other at 740-780 nm (the Pfr state). All BphPs exhibit natural photochromic behavior: they undergo reversible $\mathrm{Pfr} \rightarrow \operatorname{Pr}$ photoswitching upon 730-790 nm light irradiation and $\mathrm{Pr} \rightarrow$ Pfr photoswitching upon 630-690 nm light irradiation. Here, we term the Pfr state of the BphP-based probes the "ON" state and the Pr state the "OFF" state.

Currently, because of the absence of PA probes with NIR absorbance, whole-body molecular imaging of proteinprotein interactions (PPIs) employs bioluminescent luciferases and FPs. PPI studies utilize Förster resonance energy transfer (FRET), bioluminescence energy transfer (BRET), and bimolecular fluorescence complementation (BiFC) approaches. However, relatively small changes in the FRET and BRET signals make these techniques suboptimal for use in whole mammals. BiFC is based on the tagging of two proteins of interest, each with half of an FP. Upon interaction of the proteins, the two halves of the split FP associate with each other to form a fluorescent complex with the complemented FP, thus reporting the PPIs. Recently, we engineered several BiFC reporters from NIR FPs and demonstrated their ability to detect PPIs in mice[20]. However, NIR BiFC did not provide high spatial resolution and sensitivity in imaging PPIs in deep tumors. PPIs were also imaged in vivo using split luciferase[21] and thymidine kinase[22], resulting in bioluminescence and positron emission signals, respectively. However, these reporters require injection of substrates. Moreover, the emission of the most red-shifted split-luciferase is limited to $615 \mathrm{~nm}[23]$, and thymidine kinase's signal provides low contrast and a non-specific background in vivo.

Here, we report a PACT technique which combines three approaches, namely single-impulse panoramic PACT (SIP-PACT)[2], RS-PACT[19], and real-time detection of the photoswitching rates of genetically encoded photochromic probes. We term this combined technique RS-SIP-PACT.

\section{RESULTS}

\subsection{Characterization of DrBphP-PCM}

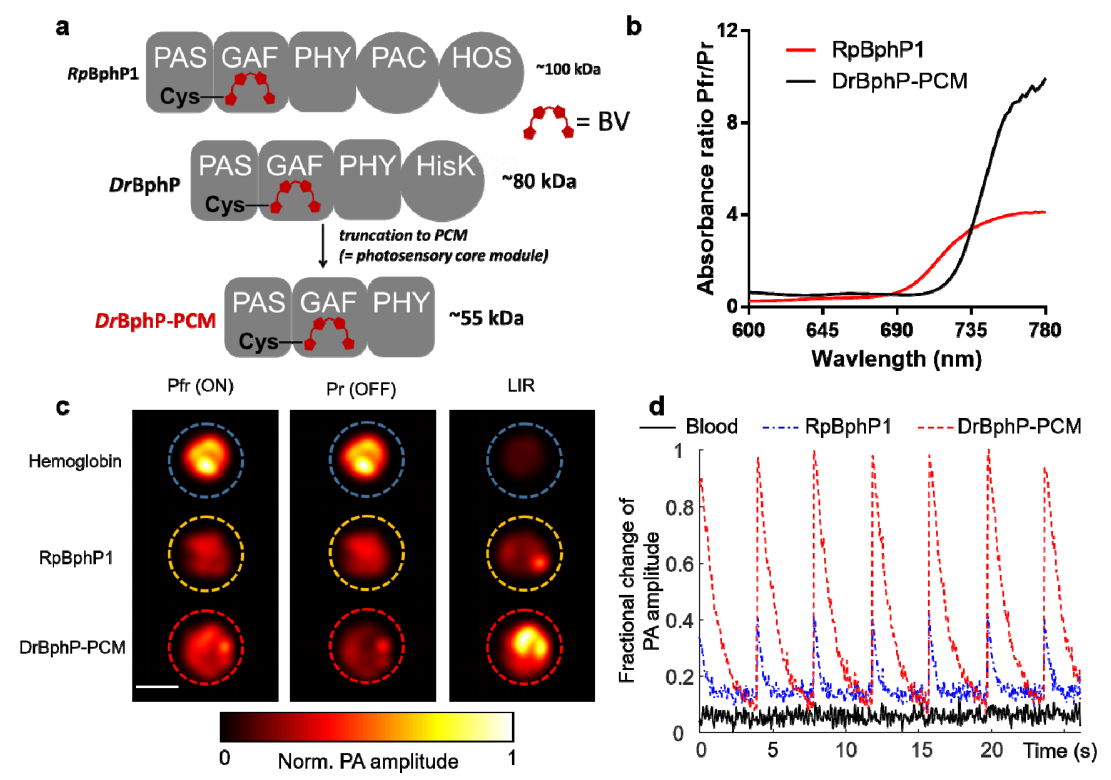

Figure 1. (a) Development of a smaller photochromic probe for differential photoacoustic imaging. (b) extinction coefficient ratios between the Pfr and Pr states of DrBphP-PCM and RpBphP1, showing the superior switching contrast of DrBphPPCM in the NIR spectral region. (c) PA images of hemoglobin, RpBphP1, and DrBphP-PCM in the Pfr (ON) state (left column) and $\operatorname{Pr}$ (OFF) state (middle column). LIR images of hemoglobin, RpBphP1, and DrBphP-PCM (right column). Scale bar, $2 \mathrm{~mm}$. (d) Fractional change of PA signals from bovine blood, U87 cells expressing either RpBphP1 or DrBphPPCM during $780 \mathrm{~nm}$ illumination, showing different photoswitching rate. 
We first measured the molar extinction coefficients for the ON states and the OFF states of DrBphP-PCM and RpBphP1. The ratios between the extinction coefficients of the ON state (Pfr form) at $780 \mathrm{~nm}$ and the OFF state (Pr form) at 630 $\mathrm{nm}$ of DrBphP-PCM and RpBphP1 were 9.9 and 4.1, respectively (Fig. 1b). We employed $780 \mathrm{~nm}$ light for PA imaging and photoswitching the BphPs to the OFF state, and used $630 \mathrm{~nm}$ light to switch the BphPs back to the ON state. The laser fluence on the sample surface at both wavelengths did not exceed $12 \mathrm{~mJ} \mathrm{~cm}^{-2}$, which is below the American National Standards Institute safety limit[24]. We next used RS-SIP-PACT to image U87 glioblastoma cells stably expressing either RpBphP1 or DrBphP-PCM. With the RS-SIP-PACT system, we imaged pure bovine blood and either RpBphP1 or DrBphP-PCM expressing U87 cells embedded in gelatin (Fig. 1c). Temporal frequency lock-in PA reconstruction (LIR) successfully separated the PA signals from two BphPs from the non-photoswitchable blood signals. The decay constants from the ON to the OFF state enabled good separation of the blood and both types of U87 cells (Fig. 1d).

\subsection{Multi-contrast RS-SIP-PACT imaging in vivo}

We used RS-SIP-PACT to image the HEK-293 cells expressing both BphPs in equimolar quantities from a single plasmid and the U87 cells expressing only DrBphP-PCM. For each measurement voxel, we reasonably assumed that the local fluence was uniform within that voxel, because the 1/e optical penetration depth for NIR light is far greater than the voxel length. Experimental results showed that the photoswitching signals from HEK-293 cells expressing both BphPs contained two decay components, while the signals from U87 cells expressing DrBphP-PCM exhibited only one decay component, regardless of local fluence. In vitro experiments showed a good separation of the two types of cells (Fig. 2a). To reliably separate the two types of tumors inside deep tissue in vivo, we applied this labeling strategy to the liver tumors. We first injected U87 cells expressing DrBphP-PCM $\left(0.5 \times 10^{6}\right)$ into the right lobe of the mouse liver and waited 5 days to allow the injected U87 cells to grow. After the waiting period, we injected HEK-293 cells expressing both BphPs $\left(8 \times 10^{6}\right)$ into the left lobe of the liver. At two hours post injection, we then imaged the tumor-bearing mouse $(\mathrm{n}=$ 3 ). The previous RS-PACT system required $1.6 \mathrm{~s}$ to form a cross-sectional image, which blurred whole-body images due to respiratory motion during data acquisition and thus reduced the detection sensitivity. In comparison, RS-SIP-PACT takes just $50 \mu \mathrm{s}$ to form a cross-sectional image, with completely negligible motion artifacts. By taking advantage of this real-time imaging capability, RS-SIP-PACT can image the decays of both BphPs while monitoring the respiratory motion. Thus, LIR can highlight the tumors, with minimized motion artifacts and high contrast (Fig. 2b, c). The signal

decays from the tumors can be modeled in the form of $g(t)=a+b \cdot e^{\left(\frac{-t}{T_{1}}\right)}+c \cdot e^{\left(\frac{-t}{T_{2}}\right)}$, where $T_{1}>T_{2}$. The signals from HEK-293 cells were fitted with two similar coefficients $b \approx c \approx 0.5$, while the signals from U87 cells were fitted with very different coefficients $b \approx 1, c \approx 0$. The HEK-293 tumors contain two different photochromic proteins, exhibiting two different decay constants in the decay process; while the U87 tumors contain only one photochromic protein, exhibiting only one decay constant in the decay process. Moreover, by analyzing the number of decay constants involved, we achieved reliable differentiation between the two tumors in deep tissue ( $\sim 9.1 \mathrm{~mm}$ beneath skin, Fig. $2 \mathrm{~d})$. 


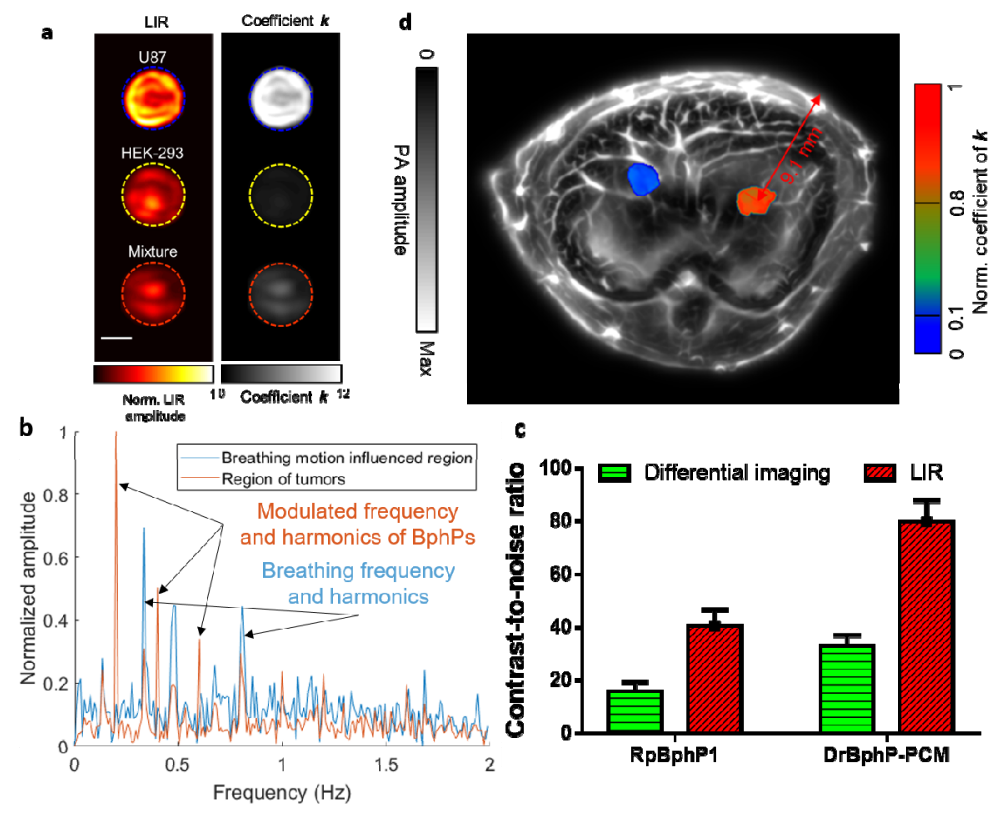

Figure 2. Separation of two types of cells at depths. (a) Separation of different types of cells expressing different BphPs in scattering media. (left) LIR images of the cells; (right) computed coefficients, $k$, of the cells, which enables a good separation of each other. (b) Temporal frequency spectra of the PA signals in the kidney tumors and the internal organs, showing both the harmonics of the illumination modulation frequency and the harmonics of the respiratory frequency. (c) The LIR method provides $\sim 2-3$-fold better CNR of tumor cells expressing either DrBphP-PCM or RpBphP1 than the differential imaging method; error bars are s.e.m. $(n=80)$, calculated based on the pixel values from regions of interest. (d) Normalized coefficient k encoded image overlaid on a conventional PACT cross-sectional image. Scale bar, $5 \mathrm{~mm}$.

\subsection{RS-SIP-PACT imaging of PPIs with DrSplit}

Using DrSplit and RS-SIP-PACT, we next longitudinally imaged PPIs in the tumors and monitored tumor metastases in the liver of mice. DrSplit-expressing MTLn3 cells $\left(1 \times 10^{6}\right)$ were first locally injected in the mouse liver. Fig. 3a shows the PACT image of the mouse liver on Day 32 after tumor cells injection but before rapamycin injection. The LIR image was overlaid on the anatomical image, but no lock-in frequency signal was detected. Then, rapamycin was injected through the tail vein $\sim 40-44 \mathrm{~h}$ before the PA imaging. The LIR PA images highlighted the photoswitchable signals from the complemented DrSplit resulting from the PPIs (Fig. 3b). And the tumor growth has been confirmed histologically (Fig. 3c). We have also demonstrated an application of DrSplit as BiFC reporter at depths in vivo. We detected the fluorescence of reconstituted DrSplit after 32 days of tumor growth upon the induction of PPIs with rapamycin (Fig. 3d).

\section{DISCUSSION}

PACT has demonstrated its capability for anatomical, functional, and molecular imaging.[25-30] Particularly, for molecular imaging, PACT is well suited to take maximum advantage of the photochromic behavior of genetically encoded probes.[31, 32] Here, we combined the advanced RS-SIP-PACT technique with two distinct BphP-based probes, DrBphP-PCM and RpBphP1, which enabled multi-contrast PA in vivo imaging with a single $780 \mathrm{~nm}$ excitation. We then designed the first BiPC reporter, DrSplit, and by combining it with RS-SIP-PACT, photoacoustically detected PPIs with high spatial resolution in deep tissues at the whole-body level in mice. These advances resulted from the photochemical and structural features of DrBphP-PCM, which are superior to those of the previously used RpBphP1, as well as from the high imaging speed and the LIR approach of RS-SIP-PACT, which provided high-sensitivity, highresolution imaging at depths beyond those achievable by pure optical imaging[33]. 


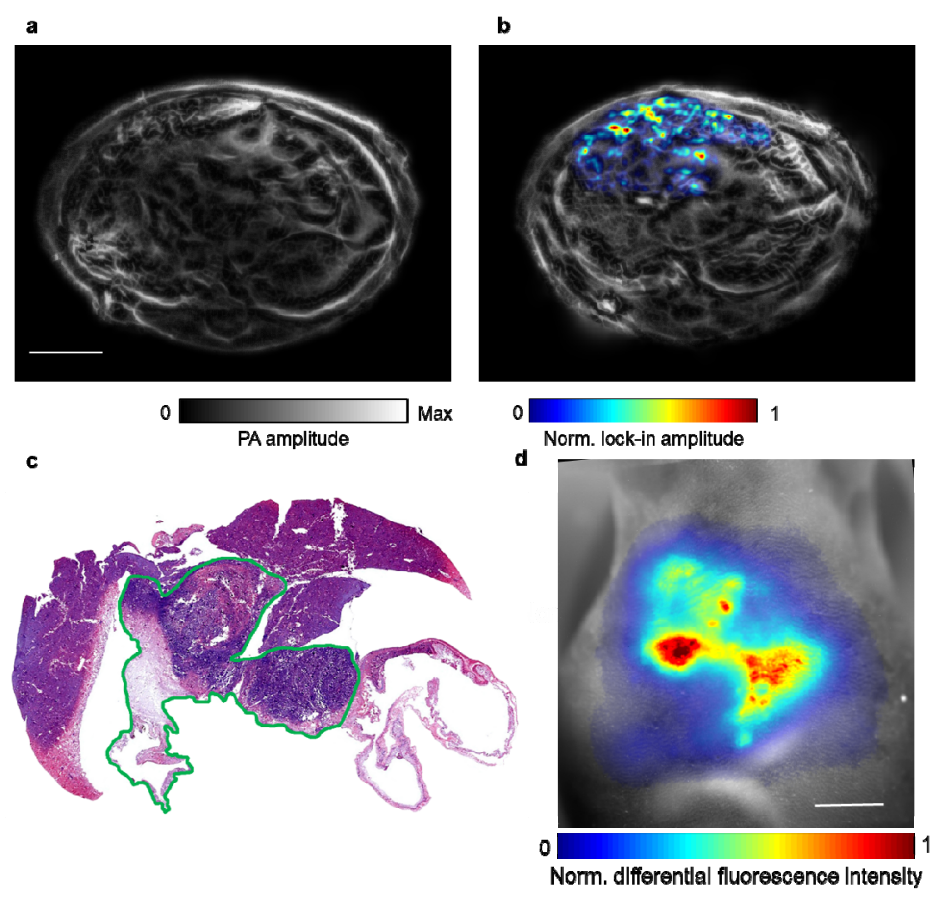

Figure 3. In vivo RS-SIP-PACT of liver tumors expressing DrSplit, showing photoacoustically detected PPIs at depth. (a) PACT image of the mouse liver on Day 32 after tumor cells injection but before rapamycin injection. The LIR image was overlaid on the anatomical image, but no lock-in frequency signal was detected. Scale bar, $5 \mathrm{~mm}$. (b) PACT image of the mouse on Day 34 after tumor cells injection (44 h after rapamycin injection). The LIR image was overlaid on the anatomical image, showing the PPI-induced complementation of DrBphP-PCM from DrSplit. (c) Representative H\&E histological image of a harvested tumorous liver, where the tumor region is bordered by the green line. (d) Fluorescence image of the mouse on Day 32 after the injection of tumor cells ( $\sim 4$ hours after rapamycin injection), the differential image (in color) was overlaid on the anatomical image (in gray). Scale bar, $5 \mathrm{~mm}$.

\section{REFERENCES}

[1] J. Yao, L. Wang, J. M. Yang et al., "High-speed label-free functional photoacoustic microscopy of mouse brain in action," Nat Methods, 12(5), 407-10 (2015).

[2] L. Li, L. Zhu, C. Ma et al., "Single-impulse Panoramic Photoacoustic Computed Tomography of Small-animal Whole-body Dynamics at High Spatiotemporal Resolution," Nat Biomed Eng, 1(5), (2017).

[3] O. T. Bruns, T. S. Bischof, D. K. Harris et al., "Next-generation in vivo optical imaging with short-wave infrared quantum dots," Nat Biomed Eng, 1, (2017).

[4] M. de Jong, J. Essers, and W. M. van Weerden, "Imaging preclinical tumour models: improving translational power," Nat Rev Cancer, 14(7), 481-493 (2014).

[5] R. Weissleder, and M. J. Pittet, "Imaging in the era of molecular oncology," Nature, 452(7187), 580-589 (2008).

[6] H.-C. Hsu, L. Li, J. Yao et al., "Dual-axis illumination for virtually augmenting the detection view of opticalresolution photoacoustic microscopy," Journal of biomedical optics, 23(7), 076001 (2018).

[7] Y. He, L. Wang, J. Shi et al., "In vivo label-free photoacoustic flow cytography and on-the-spot laser killing of single circulating melanoma cells," Scientific reports, 6, 39616 (2016).

[8] W. L. Rice, D. M. Shcherbakova, V. V. Verkhusha et al., "In vivo tomographic imaging of deep-seated cancer using fluorescence lifetime contrast," Cancer Res, 75(7), 1236-43 (2015).

[9] L. Li, C. Yeh, S. Hu et al., "Fully motorized optical-resolution photoacoustic microscopy," Optics letters, 39(7), 2117-2120 (2014).

[10] L. Zhu, L. Li, L. Gao et al., "Multiview optical resolution photoacoustic microscopy," Optica, 1(4), 217-222 (2014). 
[11] C. Yeh, L. Li, L. Zhu et al., "Dry coupling for whole-body small-animal photoacoustic computed tomography," Journal of biomedical optics, 22(4), 041017 (2017).

[12] L. Lin, J. Xia, T. T. Wong et al., "In vivo deep brain imaging of rats using oral-cavity illuminated photoacoustic computed tomography," Journal of biomedical optics, 20(1), 016019 (2015).

[13] T. P. Matthews, J. Poudel, L. Li et al., "Parameterized Joint Reconstruction of the Initial Pressure and Sound Speed Distributions for Photoacoustic Computed Tomography," SIAM Journal on Imaging Sciences, 11(2), 1560-1588 (2018).

[14] J. Poudel, T. P. Matthews, L. Li et al., "Mitigation of artifacts due to isolated acoustic heterogeneities in photoacoustic computed tomography using a variable data truncation-based reconstruction method," Journal of biomedical optics, 22(4), 041018 (2017).

[15] B. Rao, R. Zhang, L. Li et al., "Photoacoustic imaging of voltage responses beyond the optical diffusion limit," Scientific Reports, 7(1), 2560 (2017).

[16] A. P. Jathoul, J. Laufer, O. Ogunlade et al., "Deep in vivo photoacoustic imaging of mammalian tissues using a tyrosinase-based genetic reporter," Nat Photon, 9, 239-246 (2015).

[17] M. Zhou, L. Li, J. Yao et al., [Nanoparticles for Photoacoustic Imaging of Vasculature] Springer, Cham, (2017).

[18] Y. Qu, L. Li, Y. Shen et al., "Dichroism-sensitive photoacoustic computed tomography," Optica, 5(4), $495-501$ (2018).

[19] J. Yao, A. A. Kaberniuk, L. Li et al., "Multiscale photoacoustic tomography using reversibly switchable bacterial phytochrome as a near-infrared photochromic probe," Nat Methods, 13(1), 67-73 (2016).

[20] G. S. Filonov, and V. V. Verkhusha, "A near-infrared BiFC reporter for in vivo imaging of protein-protein interactions," Chem Biol, 20(8), 1078-86 (2013).

[21] K. E. Luker, L. A. Mihalko, B. T. Schmidt et al., "In vivo imaging of ligand receptor binding with Gaussia luciferase complementation," Nat Med, 18(1), 172-7 (2011).

[22] T. F. Massoud, R. Paulmurugan, and S. S. Gambhir, "A molecularly engineered split reporter for imaging protein-protein interactions with positron emission tomography," Nat Med, 16(8), 921-6 (2010).

[23] V. Villalobos, S. Naik, M. Bruinsma et al., "Dual-color click beetle luciferase heteroprotein fragment complementation assays," Chem Biol, 17(9), 1018-29 (2010).

[24] A. N. S. I. I. (ANSI), [American National Standard for Safe Use of Lasers], (2007).

[25] P. Zhang, L. Li, L. Lin et al., "High - resolution deep functional imaging of the whole mouse brain by photoacoustic computed tomography in vivo,” Journal of biophotonics, 11(1), e201700024 (2018).

[26] L. Lin, P. Hu, J. Shi et al., "Single-breath-hold photoacoustic computed tomography of the breast," Nature communications, 9(1), 2352 (2018).

[27] T. Imai, J. Shi, T. T. Wong et al., "High-throughput ultraviolet photoacoustic microscopy with multifocal excitation," Journal of biomedical optics, 23(3), 036007 (2018).

[28] L. Li, J. Xia, G. Li et al., "Label-free photoacoustic tomography of whole mouse brain structures ex vivo," Neurophotonics, 3(3), 035001 (2016).

[29] Y. S. Zhang, J. Yao, C. Zhang et al., “Optical - Resolution Photoacoustic Microscopy for Volumetric and Spectral Analysis of Histological and Immunochemical Samples," Angewandte Chemie, 126(31), 8237-8241 (2014).

[30] L. Li, L. Zhu, Y. Shen et al., "Multiview Hilbert transformation in full-ring transducer array-based photoacoustic computed tomography," Journal of biomedical optics, 22(7), 076017 (2017).

[31] J. Yao, A. A. Kaberniuk, L. Li et al., "Reversibly switchable photoacoustic tomography using a genetically encoded near-infrared phytochrome." 9708, 97082U.

[32] L. Li, L. Zhu, C. Ma et al., "Imaging small animal whole-body dynamics by single-impulse panoramic photoacoustic computed tomography." 10064, 100640M.

[33] Y. Yan, M. E. Marriott, C. Petchprayoon et al., "Optical switch probes and optical lock-in detection (OLID) imaging microscopy: high-contrast fluorescence imaging within living systems," Biochem J, 433(3), 411-22 (2011). 